\title{
PROPERNESS OF ASSOCIATED MINIMAL SURFACES
}

\author{
ANTONIO ALARCÓN AND FRANCISCO J. LÓPEZ
}

Abstract. For any open Riemann surface $\mathcal{N}$ and finite subset $\mathfrak{Z} \subset \mathbb{S}^{1}=\{z \in$ $\mathbb{C}|| z \mid=1\}$, there exist an infinite closed set $\mathfrak{Z} \mathcal{N} \subset \mathbb{S}^{1}$ containing $\mathfrak{Z}$ and a null holomorphic curve $F=\left(F_{j}\right)_{j=1,2,3}: \mathcal{N} \rightarrow \mathbb{C}^{3}$ such that the map

$$
\mathfrak{Y}: \mathfrak{Z}_{\mathcal{N}} \times \mathcal{N} \rightarrow \mathbb{R}^{2}, \quad \mathfrak{Y}(\mathfrak{v}, P)=\operatorname{Re}\left(\mathfrak{v}\left(F_{1}, F_{2}\right)(P)\right)
$$

is proper.

In particular, $\operatorname{Re}(\mathfrak{v} F): \mathcal{N} \rightarrow \mathbb{R}^{3}$ is a proper conformal minimal immersion properly projecting into $\mathbb{R}^{2} \equiv \mathbb{R}^{2} \times\{0\} \subset \mathbb{R}^{3}$, for all $\mathfrak{v} \in \mathfrak{Z}_{\mathcal{N}}$.

\section{INTRODUCTION}

Given an open Riemann surface $\mathcal{N}$, a conformal minimal immersion $X: \mathcal{N} \rightarrow \mathbb{R}^{3}$ is said to be flux-vanishing if the conjugate immersion $X^{*}: \mathcal{N} \rightarrow \mathbb{R}^{3}$ is well defined or, equivalently, if $X$ is the real part of a null holomorphic curve $F: \mathcal{N} \rightarrow \mathbb{C}^{3}$ (see Definition 2.3). In this case, the family of isometric associated minimal immersions $X_{\mathfrak{v}} \equiv \operatorname{Re}(\mathfrak{v} F): \mathcal{N} \rightarrow \mathbb{R}^{3}, \mathfrak{v} \in \mathbb{S}^{1}=\{z \in \mathbb{C}|| z \mid=1\}$, is well defined. Notice that $X=X_{1}$ and recall that $X^{*}=X_{-\imath}, \imath=\sqrt{-1}$.

The aim of this paper is to study the interplay between topological properness and associated minimal surfaces. Not so many years ago it was a general thought that properness strongly influences the underlying conformal structure of minimal surfaces in $\mathbb{R}^{3}$. In this line, Schoen and Yau asked whether there exist hyperbolic minimal surfaces in $\mathbb{R}^{3}$ properly projecting into $\mathbb{R}^{2} \equiv \mathbb{R}^{2} \times\{0\} \subset \mathbb{R}^{3}[\mathrm{SY}$, p. 18]. A complete answer to this question can be found in [AL1, where examples with arbitrary conformal structure and flux map are shown.

On the other hand, any flux-vanishing minimal surface all of whose associated surfaces uniformly properly project into $\mathbb{R}^{2}$ is parabolic; see Proposition 4.3. This suggests a correlation between the properness of associated surfaces and the conformal structure of minimal surfaces. The following questions arise:

(Q1) Do there exist hyperbolic flux-vanishing minimal surfaces $S$ such that both $S$ and its conjugate surface $S^{*}$ properly project into $\mathbb{R}^{2}$ ?

(Q2) More generally, how many associated surfaces of a hyperbolic flux-vanishing minimal surface can properly project into $\mathbb{R}^{2}$ ?

Received by the editors March 7, 2012.

2010 Mathematics Subject Classification. Primary 53A10; Secondary 32H02, 53C42.

Key words and phrases. Null holomorphic curves, associated minimal surfaces.

The first author was supported by Vicerrectorado de Política Científica e Investigación de la Universidad de Granada and was partially supported by MCYT-FEDER grants MTM2007-61775 and MTM2011-22547, Junta de Andalucía Grant P09-FQM-5088, and the grant PYR-2012-3 CEI BioTIC GENIL (CEB09-0010) of the MICINN CEI Program.

The second author was partially supported by MCYT-FEDER research projects MTM200761775 and MTM2011-22547, and Junta de Andalucía Grant P09-FQM-5088. 
Motivated by the above questions, this paper deals with those subsets $\mathfrak{Z} \subset \mathbb{S}^{1}$ allowing proper projections in a uniform way, according to the following.

Definition 1.1. A closed subset $\mathfrak{Z} \subset \mathbb{S}^{1}$ is said to be a projector set for an open Riemann surface $\mathcal{N}$ if there exists a null holomorphic curve $F=\left(F_{j}\right)_{j=1,2,3}: \mathcal{N} \rightarrow$ $\mathbb{C}^{3}$ such that the map

$$
\mathfrak{Y}: \mathfrak{Z} \times \mathcal{N} \rightarrow \mathbb{R}^{2}, \quad \mathfrak{Y}(\mathfrak{v}, P)=\operatorname{Re}\left(\mathfrak{v}\left(F_{1}, F_{2}\right)(P)\right),
$$

is proper.

Moreover, $\mathfrak{Z}$ is said to be a universal projector set if it is a projector set for any open Riemann surface.

If $\mathfrak{Z}$ is a projector set for $\mathcal{N}$ and $F$ is as in Definition 1.1, then $\operatorname{Re}(\mathfrak{v} F): \mathcal{N} \rightarrow \mathbb{R}^{3}$ is a proper conformal minimal immersion in $\mathbb{R}^{3}$ which properly projects into $\mathbb{R}^{2}$, for all $\mathfrak{v} \in \mathfrak{Z}$.

One can easily check that if $\mathfrak{Z} \subset \mathbb{S}^{1}$ is a projector set for $\mathcal{N}$, then so are $\mathfrak{v} \mathfrak{Z}$ for all $\mathfrak{v} \in \mathbb{S}^{1}, \mathfrak{Z} \cup(-\mathfrak{Z})$, and any closed subset of $\mathfrak{Z}$.

Pirola's results $\mathrm{Pi}$ imply that $\mathbb{S}^{1}$ is a projector set for any parabolic Riemann surface of finite topology (see also $[\mathrm{LO})$. On the other hand, $\{1\}$ is a universal projector set [AL1], whereas Proposition 4.3 in this paper shows that $\mathbb{S}^{1}$ is not. In this line we have obtained the following.

Main Theorem. For any finite subset $\mathfrak{Z} \subset \mathbb{S}^{1}$ and any open Riemann surface $\mathcal{N}$, there exists an infinite projector set $\mathfrak{Z}_{\mathcal{N}}$ for $\mathcal{N}$ containing $\mathfrak{Z}$.

In particular, $\mathfrak{Z}$ is a universal projector set.

As a corollary, for any open Riemann surface $\mathcal{N}$ and finite set $\mathfrak{Z} \subset \mathbb{S}^{1}$, there exist an infinite subset $\mathfrak{Z}_{\mathcal{N}} \subset \mathbb{S}^{1}$ containing $\mathfrak{Z}$ and a flux-vanishing conformal minimal immersion $X: \mathcal{N} \rightarrow \mathbb{R}^{3}$ such that $X_{\mathfrak{v}}$ properly projects into $\mathbb{R}^{2}$ for all $\mathfrak{v} \in \mathfrak{Z}_{\mathcal{N}}$. This particularly answers (Q1) in the positive and enlightens us about (Q2).

It is not hard to check that if $\mathbb{S}^{1}$ is a projector set for an open Riemann surface $\mathcal{N}$, then $\mathcal{N}$ is parabolic (see Proposition 4.3). Furthermore, if $\mathcal{N}$ is of finite topology and $F: \mathcal{N} \rightarrow \mathbb{C}^{3}$ is a null holomorphic curve such that the map $\mathfrak{Y}: \mathbb{S}^{1} \times \mathcal{N} \rightarrow$ $\mathbb{R}^{2}, \mathfrak{Y}(\mathfrak{v}, P)=\operatorname{Re}\left(\mathfrak{v}\left(F_{1}, F_{2}\right)(P)\right)$, is proper, then $F$ has finite total curvature (see Corollary 4.4). Connecting with the classical Sullivan's conjecture for properly immersed minimal surfaces in $\mathbb{R}^{3}$, see [Mo], the following question remains open:

(Q3) Let $\mathcal{N}$ be an open Riemann surface of finite topology, and assume there exists a null holomorphic curve $F: \mathcal{N} \rightarrow \mathbb{C}^{3}$ such that the map

$$
\mathbb{S}^{1} \times \mathcal{N} \rightarrow \mathbb{R}^{3}, \quad(\mathfrak{v}, P) \mapsto \operatorname{Re}(\mathfrak{v} F(P)),
$$

is proper. Must $\mathcal{N}$ be of parabolic conformal type? Even more, must $F$ be of finite total curvature?

Our main tools come from approximation results for minimal surfaces and null holomorphic curves developed by the authors in AL1, AL2.

\section{Preliminaries}

Denote by $\|\cdot\|$ the Euclidean norm in $\mathbb{K}^{n}$, where $\mathbb{K}=\mathbb{R}$ or $\mathbb{C}$. For any compact topological space $K$ and continuous map $f: K \rightarrow \mathbb{K}^{n}$, denote by

$$
\|f\|_{0, K}=\max \{\|f(p)\| \mid p \in K\}
$$

the maximum norm of $f$ on $K$. 
Given an $n$-dimensional topological manifold $M$, we denote by $\partial M$ the $(n-1)$ dimensional topological manifold determined by its boundary points. For any $A \subset$ $M, A^{\circ}$ and $\bar{A}$ will denote the interior and the closure of $A$ in $M$, respectively. Open connected subsets of $M-\partial M$ will be called domains, and those proper topological subspaces of $M$ being $n$-dimensional manifolds with boundary are said to be regions. If $M$ is a topological surface, $M$ is said to be open if it is non-compact and $\partial M=\emptyset$.

\subsection{Riemann surfaces.}

Remark 2.1. Throughout this paper $\mathcal{N}$ will denote a fixed but arbitrary open Riemann surface, and $\sigma_{\mathcal{N}}^{2}$ a conformal Riemannian metric on $\mathcal{N}$.

The key tool in this paper is a Mergelyan type approximation result by null holomorphic curves in $\mathbb{C}^{3}$ (see Lemma 2.6 below and [AL1, AL2]). This subsection and the next one are devoted to introducing the necessary notation for a good understanding of this result.

A Jordan arc in $\mathcal{N}$ is said to be analytical if it is contained in an open analytical Jordan $\operatorname{arc}$ in $\mathcal{N}$.

Classically, a compact region $A \subset \mathcal{N}$ is said to be Runge if $\mathcal{N}-A$ has no relatively compact components in $\mathcal{N}$ or, equivalently, if the inclusion map $\mathrm{i}_{A}: A \hookrightarrow \mathcal{N}$ induces a group monomorphism $\left(\mathrm{i}_{A}\right)_{*}: \mathcal{H}_{1}(A, \mathbb{Z}) \rightarrow \mathcal{H}_{1}(\mathcal{N}, \mathbb{Z})$, where $\mathcal{H}_{1}(\cdot, \mathbb{Z})$ means first homology group with integer coefficients. More generally, an arbitrary subset $A \subset \mathcal{N}$ is said to be Runge if $\left(\mathrm{i}_{A}\right)_{*}: \mathcal{H}_{1}(A, \mathbb{Z}) \rightarrow \mathcal{H}_{1}(\mathcal{N}, \mathbb{Z})$ is injective. In this case we identify the groups $\mathcal{H}_{1}(A, \mathbb{Z})$ and $\left(\mathrm{i}_{A}\right)_{*}\left(\mathcal{H}_{1}(A, \mathbb{Z})\right) \subset \mathcal{H}_{1}(\mathcal{N}, \mathbb{Z})$ via $\left(\mathrm{i}_{A}\right)_{*}$ and consider $\mathcal{H}_{1}(A, \mathbb{Z}) \subset \mathcal{H}_{1}(\mathcal{N}, \mathbb{Z})$.

Given an open subset $W \subset \mathcal{N}$, we denote by

- $\mathcal{F}_{\mathfrak{h}}(W)$ the space of holomorphic functions on $W$, and

- $\Omega_{\mathfrak{h}}(W)$ the space of holomorphic 1-forms on $W$.

The following definition is crucial in our arguments; see Figure 2.1.

Definition 2.2. A compact subset $S \subset \mathcal{N}$ is said to be admissible if and only if

- $M_{S}:=\overline{S^{\circ}}$ is a finite collection of pairwise disjoint compact regions in $\mathcal{N}$ with $\mathcal{C}^{0}$ boundary,

- $C_{S}:=\overline{S-M_{S}}$ consists of a finite collection of pairwise disjoint analytical Jordan arcs,

- any component $\alpha$ of $C_{S}$ with an endpoint $P \in M_{S}$ admits an analytical extension $\beta$ in $\mathcal{N}$ such that the unique component of $\beta-\alpha$ with endpoint $P$ lies in $M_{S}$, and

- $S$ is Runge.

Let $S$ be an admissible subset of $\mathcal{N}$.

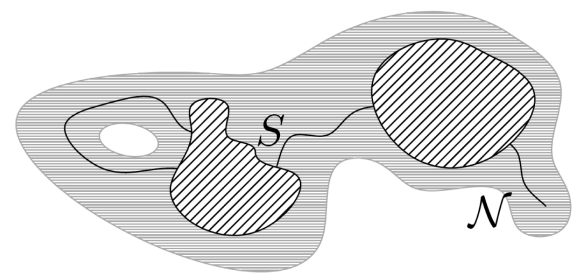

Figure 2.1. An admissible subset $S$ of $\mathcal{N}$ 
A (complex) 1-form $\theta$ on $S$ is said to be of type $(1,0)$ if for any conformal chart $(U, z)$ in $\mathcal{N},\left.\theta\right|_{U \cap S}=h(z) d z$ for some function $h: U \cap S \rightarrow \mathbb{C}$. An $n$ tuple $\Lambda=\left(\theta_{1}, \ldots, \theta_{n}\right)$, where $\theta_{j}$ is a $(1,0)$-type 1 -form for all $j$, is said to be an $n$-dimensional vectorial $(1,0)$-form on $S$. The space of continuous $n$-dimensional (1,0)-forms on $S$ will be endowed with the $\mathcal{C}^{0}$ topology induced by the norm

$$
\|\Lambda\|_{0, S}:=\left\|\frac{\Lambda}{\sigma_{\mathcal{N}}}\right\|_{0, S}=\max _{S}\left\{\left(\sum_{j=1}^{n}\left|\frac{\theta_{j}}{\sigma_{\mathcal{N}}}\right|^{2}\right)^{1 / 2}\right\}
$$

(see Remark 2.1).

We denote by

- $\mathcal{F}_{\mathfrak{h}}(S)$ the space of continuous functions $f: S \rightarrow \mathbb{C}$ which are holomorphic on an open neighborhood of $M_{S}$ in $\mathcal{N}$ and

- $\Omega_{\mathfrak{h}}(S)$ the space of 1 -forms $\theta$ of type $(1,0)$ on $S$ such that $\theta / \vartheta \in \mathcal{F}_{\mathfrak{h}}(S)$ for any nowhere-vanishing holomorphic 1 -form $\vartheta$ on $\mathcal{N}$ (the existence of such a $\vartheta$ was proved in GN]; a modern proof can be found in [AFL]).

Smoothness of functions and 1-forms on admissible sets are defined as follows:

- A function $f \in \mathcal{F}_{\mathfrak{h}}(S)$ is said to be smooth if $\left.f\right|_{M_{S}}$ admits a smooth extension $f_{0}$ to a domain $W \subset \mathcal{N}$ containing $M_{S}$, and for any component $\alpha$ of $C_{S}$ and any open analytical Jordan $\operatorname{arc} \beta$ in $\mathcal{N}$ containing $\alpha, f$ admits a smooth extension $f_{\beta}$ to $\beta$ satisfying that $\left.f_{\beta}\right|_{W \cap \beta}=\left.f_{0}\right|_{W \cap \beta}$.

- A 1-form $\theta \in \Omega_{\mathfrak{h}}(S)$ is said to be smooth if $\theta / \vartheta \in \mathcal{F}_{\mathfrak{h}}(S)$ is smooth, for any nowhere-vanishing holomorphic 1-form $\vartheta$ on $\mathcal{N}$.

Given a smooth function $f \in \mathcal{F}_{\mathfrak{h}}(S)$, the differential $d f$ of $f$ is given by

$$
\left.d f\right|_{M_{S}}=d\left(\left.f\right|_{M_{S}}\right) \text { and }\left.d f\right|_{\alpha \cap U}=\left.(f \circ \alpha)^{\prime}(x) d z\right|_{\alpha \cap U},
$$

where $(U, z=x+\imath y), \imath=\sqrt{-1}$, is a conformal chart on $\mathcal{N}$ such that $\alpha \cap U=$ $z^{-1}(\mathbb{R} \cap z(U))$. Notice that $d f \in \Omega_{\mathfrak{h}}(S)$ and is smooth as well.

Finally, the $\mathcal{C}^{1}$-norm on $S$ of a smooth $f \in \mathcal{F}_{\mathfrak{h}}(S)$ is defined by

$$
\|f\|_{1, S}=\max \left\{\|f(P)\|+\left\|\frac{d f}{\sigma_{\mathcal{N}}}(P)\right\| \mid P \in S\right\} .
$$

In a similar way, one can define the notions of smoothness, (vectorial) differential and $\mathcal{C}^{1}$-norm for functions $f: S \rightarrow \mathbb{C}^{k}, k \in \mathbb{N}$.

2.2. Null curves in $\mathbb{C}^{3}$. Throughout this paper we adopt column notation for both vectors and matrices of linear transformations in $\mathbb{C}^{3}$. As usual, $(\cdot)^{T}$ means transpose matrix. The following operators are strongly related to the geometry of $\mathbb{C}^{3}$ and null curves. We denote by

- $\ll \cdot, \cdot \gg: \mathbb{C}^{3} \times \mathbb{C}^{3} \rightarrow \mathbb{C}, \ll u, v \gg=\bar{u}^{T} \cdot v$ the usual Hermitian inner product of $\mathbb{C}^{3}$,

- $\langle\cdot, \cdot\rangle=\operatorname{Re}(\ll \cdot, \cdot \gg): \mathbb{C}^{3} \times \mathbb{C}^{3} \rightarrow \mathbb{R}$ the Euclidean scalar product of $\mathbb{C}^{3} \equiv \mathbb{R}^{6}$, and

- $\prec \cdot, \succ \succ: \mathbb{C}^{3} \times \mathbb{C}^{3} \rightarrow \mathbb{C}$ the complex symmetric bilinear 1-form given by $\prec u, v \succ=u^{T} \cdot v$.

We also set $\ll V \gg^{\perp}=\left\{v \in \mathbb{C}^{3} \mid \ll u, v \gg=0 \forall u \in V\right\},\langle V\rangle^{\perp}=\left\{v \in \mathbb{C}^{3} \mid\langle u, v\rangle=\right.$ $0 \forall u \in V\}$ and $\prec V \succ^{\perp}=\left\{v \in \mathbb{C}^{3} \mid \prec u, v \succ=0 \forall u \in V\right\}$ for any $V \subset \mathbb{C}^{3}$. Notice that $\prec \bar{u} \succ^{\perp}=\ll u \gg^{\perp} \subset\langle u\rangle^{\perp}$ for all $u \in \mathbb{C}^{3}$, and the equality holds if and only if $u=\overrightarrow{0}:=(0,0,0)^{T}$. 
A basis $\left\{u_{1}, u_{2}, u_{3}\right\}$ of $\mathbb{C}^{3}$ is said to be $\prec \cdot, \succ \succ$-conjugate if $\prec u_{j}, u_{k} \succ=\delta_{j k}$, $j, k \in\{1,2,3\}$. Likewise, we define the notion of a $\prec \cdot, \cdot \succ$-conjugate basis of a complex subspace $U$, provided that $\prec \cdot,\left.\succ\right|_{U \times U}$ is a non-degenerate complex bilinear form.

We denote by $\mathcal{O}(3, \mathbb{C})$ the complex orthogonal group $\left\{A \in \mathcal{M}_{3}(\mathbb{C}) \mid A^{T} A=\right.$ $\left.I_{3}\right\}$, that is to say: the group of matrices whose column vectors determine a $\prec \cdot, \succ$-conjugate basis of $\mathbb{C}^{3}$. We also denote by $A: \mathbb{C}^{3} \rightarrow \mathbb{C}^{3}$ the complex linear transformation induced by $A \in \mathcal{O}(3, \mathbb{C})$. Observe that

$$
\prec A u, A v \succ=\prec u, v \succ \quad \text { and } \quad \ll A u, \bar{A} v \gg=\ll u, v \gg, \quad \forall u, v \in \mathbb{C}^{3}, A \in \mathcal{O}(3, \mathbb{C}) \text {. }
$$

A vector $u \in \mathbb{C}^{3}-\{\overrightarrow{0}\}$ is said to be null if $\prec u, u \succ=0$. We denote

$$
\Theta=\left\{u \in \mathbb{C}^{3}-\{\overrightarrow{0}\} \mid u \text { is null }\right\} .
$$

Let $M$ be an open Riemann surface.

Definition 2.3. A holomorphic map $F: M \rightarrow \mathbb{C}^{3}$ is said to be a null curve if $\prec d F, d F \succ=0$ and $\ll d F, d F \gg$ never vanishes on $M$.

Conversely, given an exact holomorphic vectorial 1-form $\Phi$ on $M$ satisfying that $\prec \Phi, \Phi \succ=0$ and $\ll \Phi, \Phi \gg$ never vanishes on $M$, then the map $F: M \rightarrow \mathbb{C}^{3}$, $F(P)=\int^{P} \Phi$, defines a null curve in $\mathbb{C}^{3}$. In this case $\Phi=d F$ is said to be the Weierstrass representation of $F$.

A null curve $F: M \rightarrow \mathbb{C}^{3}$ is said to be non-flat if $F(M)$ is contained in no null complex straight line.

Definition 2.4. Given a proper subset $M \subset \mathcal{N}$, we denote by $\mathrm{N}(M)$ the space of maps $F: M \rightarrow \mathbb{C}^{3}$ extending as a null curve to an open neighborhood of $M$ in $\mathcal{N}$.

The following definition deals with the notion of null curves on admissible subsets.

Definition 2.5. Let $S \subset \mathcal{N}$ be an admissible subset. A smooth map $F \in \mathcal{F}_{\mathfrak{h}}(S)^{3}$ is said to be a generalized null curve in $\mathbb{C}^{3}$ if it satisfies the following properties:

- $\left.F\right|_{M_{S}} \in \mathrm{N}\left(M_{S}\right)$,

- $\prec d F, d F \succ=0$ and $\ll d F, d F \gg$ never vanishes on $S$.

If $F$ is a null curve and $A \in \mathcal{O}(3, \mathbb{C})$, then $A \circ F$ is a null curve as well. The same holds for generalized null curves.

The following Mergelyan type result for null curves is a key tool in this paper. It will be used to approximate generalized null curves by null curves which are defined on larger domains.

Lemma 2.6 (AL1, AL2 ). Let $S \subset \mathcal{N}$ be admissible and connected, let $F=$ $\left(F_{j}\right)_{j=1,2,3} \in \mathcal{F}_{\mathfrak{h}}(S)^{3}$ be a generalized null curve in $\mathbb{C}^{3}$, and let $W \subset \mathcal{N}$ be a domain of finite topology containing $S$ such that $\left(\mathrm{i}_{S}\right)_{*}: \mathcal{H}_{1}(S, \mathbb{Z}) \rightarrow \mathcal{H}_{1}(W, \mathbb{Z})$ is an isomorphism, where $\mathrm{i}_{S}: S \rightarrow W$ denotes the inclusion map.

Then, for any $\epsilon>0$, there exists $H=\left(H_{j}\right)_{j=1,2,3} \in \mathrm{N}(W)$ such that $\|H-F\|_{1, S}<\epsilon$. Moreover, one can choose $H_{3}=F_{3}$ provided that $F_{3} \in \mathcal{F}_{\mathfrak{h}}(W)$ and $d F_{3}$ never vanishes on $C_{S}$. 


\section{MAIN LEMMA}

Let us begin by introducing some notation.

Let $\mathfrak{Z}=\left\{\mathfrak{v}_{1}, \ldots, \mathfrak{v}_{\mathfrak{n}}\right\} \subset \mathbb{S}^{1}$ with cardinal number $\mathfrak{n} \in \mathbb{N}$. Let $u=\left(z_{1}, z_{2}, z_{3}\right) \in \mathbb{C}^{3}$, let $\mathfrak{v} \in \mathfrak{Z}$, let $X$ be a topological space, let $K \subset X$ be a compact subset, and let $F=\left(F_{1}, F_{2}, F_{3}\right): X \rightarrow \mathbb{C}^{3}$ be a continuous map. We denote

- $u^{\mathfrak{v}}=\operatorname{Re}\left[\mathfrak{v}\left(z_{1}, z_{2}\right)\right] \in \mathbb{R}^{2}$

- $F^{\mathfrak{v}}=\operatorname{Re}\left[\mathfrak{v}\left(F_{1}, F_{2}\right)\right]: X \rightarrow \mathbb{R}^{2}$, and $F^{\mathfrak{Z}}=\left(F^{\mathfrak{v}_{j}}\right)_{j=1, \ldots, \mathfrak{n}}: X \rightarrow \mathbb{R}^{2 \mathfrak{n}}$,

- $\llbracket F^{\mathfrak{3}} \rrbracket: X \rightarrow \mathbb{R}, \llbracket F^{\mathfrak{3}} \rrbracket(P)=\min \left\{\left\|F^{\mathfrak{v}}(P)\right\| \mid \mathfrak{v} \in \mathfrak{Z}\right\}$, and $\llbracket F^{\mathfrak{3}} \rrbracket_{K}=\min _{K} \llbracket F^{\mathfrak{3}} \rrbracket$.

The following technical result is the core of our construction.

Lemma 3.1. Let $M, V$ be two admissible compact regions in $\mathcal{N}$ such that $M \subset V^{\circ}$. Let $\mathfrak{Z} \subset \mathbb{S}^{1}$ be a finite subset with cardinal number $\mathfrak{n}$, and consider $F \in \mathrm{N}(M)$ and $\delta>0$ such that

$$
\llbracket F^{\mathfrak{3}} \rrbracket_{\partial M}>\delta .
$$

Then, for any $\epsilon>0$ and any $\kappa>\delta$, there exists $\hat{F} \in \mathrm{N}(V)$ satisfying

(L1) $\|\hat{F}-F\|_{1, M}<\epsilon$,

(L2) $\llbracket \hat{F}^{3} \rrbracket_{V-M^{\circ}}>\delta / \mathfrak{n}$, and

(L3) $\llbracket \hat{F}^{\mathfrak{Z}} \rrbracket \partial V>\kappa$.

Roughly speaking, the lemma asserts that a finite family of compact associated minimal surfaces whose boundaries lie outside a cylinder in $\mathbb{R}^{3}$ can be stretched near the boundary in such a way that the boundaries of the new associated surfaces lie outside a larger parallel cylinder. In this process the topology and even the conformal structure of the arising family can be chosen arbitrarily large. See Figure 3.1

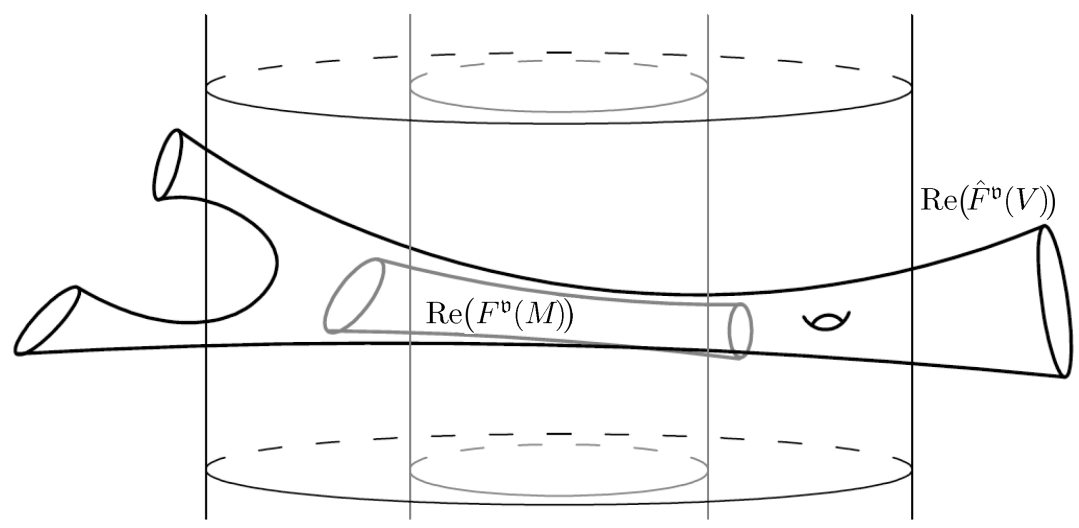

Figure 3.1. Lemma 3.1

The proof of Lemma 3.1 goes by induction on (minus) the Euler characteristic of $V-M^{\circ}$ (notice that $\left.-\chi\left(V-M^{\circ}\right) \geq 0\right)$. The hard part of the proof is the basis of the induction, which roughly goes as follows.

First we split $\partial M$ into a suitable family of small Jordan $\operatorname{arcs} \alpha_{i, j}$ (see properties (a1), (a2), and (a3) below), and assign to each of them a complex direction $e_{i, j}$ in $\mathbb{C}^{3}$ (see (3.3)). The splitting is made so that deformations of $F$ around $\alpha_{i, j}$ preserving 
the direction $e_{i, j}$, keep the boundaries of all the $\mathfrak{Z}$-associated minimal surfaces outside the cylinder of radius $\delta / \mathfrak{n}$. This choice is possible by basic trigonometry; see Claim 3.2 ,

In a second step, we construct an admissible set $S$ by attaching to $M$ a family of Jordan $\operatorname{arcs} r_{i, j}$ connecting $\alpha_{i, j}$ and $\partial V$. Then, we approximate $F$ on $M$ by a null curve $H \in \mathrm{N}(V)$ formally satisfying the theses of the lemma on $S$; see items (c1) to $(\mathrm{c} 4)$.

Finally, we modify $H$ hardly on $S$ and strongly on $V-S$ in a recursive way to obtain the null curve $\hat{F} \in \mathrm{N}(V)$ which proves the basis of the induction; see Claim 3.4. This deformation pushes the boundaries of the $\mathfrak{Z}$-associated surfaces of $H(V)$ outside the cylinder of radius $\kappa$. Furthermore, this process hardly modifies the $e_{i, j}$-coordinate of $H$ on the connected component $\Omega_{i, j}$ of $V-S$ with $\alpha_{i, j} \subset \partial \Omega_{i, j}$; see (f2). Therefore, the $\mathfrak{Z}$-associated surfaces of the arising null curve $\hat{F}\left(V-M^{\circ}\right)$ lie outside the cylinder of radius $\delta / \mathfrak{n}$.

For the inductive step we reason as follows. If $-\chi\left(V-M^{\circ}\right)=n \in \mathbb{N}$, we use Lemma 2.6 as a bridge principle for null curves to obtain a region $U$ with $M \subset U^{\circ} \subset U \subset V^{\circ}$ and $-\chi\left(V-U^{\circ}\right)=n-1$, and a null curve $H \in \mathrm{N}(U)$ which approximates $F$ on $M$ and satisfies $\llbracket H^{\mathfrak{Z}} \rrbracket_{\partial M}>\delta$. Then, we finish by applying the induction hypothesis.

3.1. Basis of the induction. Let us show that Lemma 3.1 holds in the particular instance $\chi\left(V-M^{\circ}\right)=0$.

Up to slightly deforming $F$ (use Lemma 2.6), we can suppose that $F$ is non-flat.

Since $M \subset V^{\circ}$ and $V^{\circ}-M$ has no bounded components in $V^{\circ}$, then $V-$ $M^{\circ}=\bigcup_{i=1}^{k} \mathcal{A}_{i}$, where $\mathcal{A}_{1}, \ldots, \mathcal{A}_{k}$ are pairwise disjoint compact annuli. Write $\partial \mathcal{A}_{i}=\alpha_{i} \cup \beta_{i}$, where $\alpha_{i} \subset \partial M$ and $\beta_{i} \subset \partial V$ for all $i$.

Denote by $B(r)$ the 2-dimensional Euclidean ball $\left\{p \in \mathbb{R}^{2} \mid\|p\|<r\right\}$ for any $r>0$.

Label $\Delta=\left(\mathbb{R}^{2}-\overline{B(\delta)}\right)^{\mathfrak{n}} \subset \mathbb{R}^{2 \mathfrak{n}}$, and for any $x \in \Delta$ choose a vectorial line $L_{x} \subset \mathbb{R}^{2}$ and an open neighborhood $U_{x}$ of $x$ in $\Delta$ such that

$$
\left(q_{j}+L_{x}\right) \cap \overline{B(\delta / \mathfrak{n})}=\emptyset, \quad \forall\left(q_{1}, \ldots, q_{\mathfrak{n}}\right) \in U_{x} ;
$$

see Figure 3.2. The existence of $L_{x}$ and $U_{x}, x \in \Delta$, follows straightforwardly from the following.

Claim 3.2. For any $x_{1}, \ldots, x_{\mathfrak{n}} \in \mathbb{R}^{2}-\overline{B(1)}$, there exists a vectorial line $L \subset \mathbb{R}^{2}$ depending on $x_{1}, \ldots, x_{\mathfrak{n}}$, such that

$$
\left(x_{j}+L\right) \cap \overline{B(1 / \mathfrak{n})}=\emptyset, \quad j=1, \ldots, \mathfrak{n} .
$$

Proof. Label $W_{j}=\left\{e^{\imath t} \frac{x_{j}}{\left\|x_{j}\right\|} \mid t \in\right]-\frac{\pi}{2 \mathfrak{n}}, \frac{\pi}{2 \mathfrak{n}}[\}$ for all $j=1, \ldots, \mathfrak{n}$, and take $x_{0} \in$ $\mathbb{S}^{1}-\left(\bigcup_{j=1}^{\mathfrak{n}} W_{j}\right)$. Setting $L=\left\{t x_{0} \mid t \in \mathbb{R}\right\}$, elementary trigonometry gives that $\left(\frac{x_{j}}{\left\|x_{j}\right\|}+L\right) \cap B\left(\sin \left(\frac{\pi}{2 \mathfrak{n}}\right)\right)=\emptyset, j=1, \ldots, \mathfrak{n}$. Since $\left\|x_{j}\right\|>1$ for all $j$ and $\sin \left(\frac{\pi}{2 \mathfrak{n}}\right)>\frac{1}{\mathfrak{n}}$, we are done.

For each $n \in \mathbb{N}$ denote by $\mathbb{Z}_{n}=\{0, \ldots, n-1\}$ the additive cyclic group of integers modulus $n$. Since $\mathcal{U}:=\left\{U_{x} \mid x \in \Delta\right\}$ is an open covering of the compact set $F^{\mathfrak{Z}}(\partial M) \subset \Delta($ see (3.1) $)$, there exist $m \in \mathbb{N}, m \geq 3$, and a collection $\left\{\alpha_{i, j} \mid(i, j) \in\right.$ $\left.\{1, \ldots, k\} \times \mathbb{Z}_{m}\right\}$ such that for each $i \in\{1, \ldots, k\}$,

(a1) $\bigcup_{j=1}^{m} \alpha_{i, j}=\alpha_{i}$, 


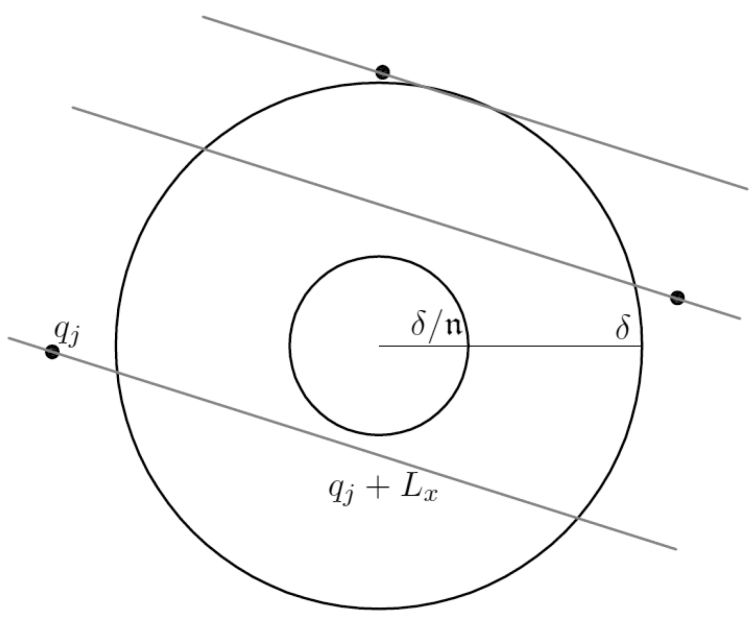

Figure 3.2. The vectorial line $L_{x} \subset \mathbb{R}^{2}$

(a2) $\alpha_{i, j}$ and $\alpha_{i, j+1}$ have a common endpoint $Q_{i, j}$ and are otherwise disjoint for all $j \in \mathbb{Z}_{m}$, and

(a3) there exists $U_{i, j} \in \mathcal{U}$ such that $F^{\mathfrak{Z}}\left(\alpha_{i, j}\right) \subset U_{i, j}$, for all $j \in \mathbb{Z}_{m}$.

If $U_{i, j}=U_{x_{i, j}}$ for $x_{i, j} \in \Delta$, for simplicity we write $L_{i, j}=L_{x_{i, j}}$ for all $(i, j) \in$ $\{1, \ldots, k\} \times \mathbb{Z}_{m}$.

Let $\left\{r_{i, j} \mid j \in \mathbb{Z}_{m}\right\}$ be a collection of pairwise disjoint analytical Jordan arcs in $\mathcal{A}_{i}$ such that $r_{i, j}$ has initial point $Q_{i, j} \in \alpha_{i}$, final point $P_{i, j} \in \beta_{i}$, and $r_{i, j}$ is otherwise disjoint from $\partial \mathcal{A}_{i}$ for all $i$ and $j$. Without loss of generality, assume that $S=M \cup\left(\bigcup_{i, j} r_{i, j}\right)$ is admissible. See Figure 3.3 .

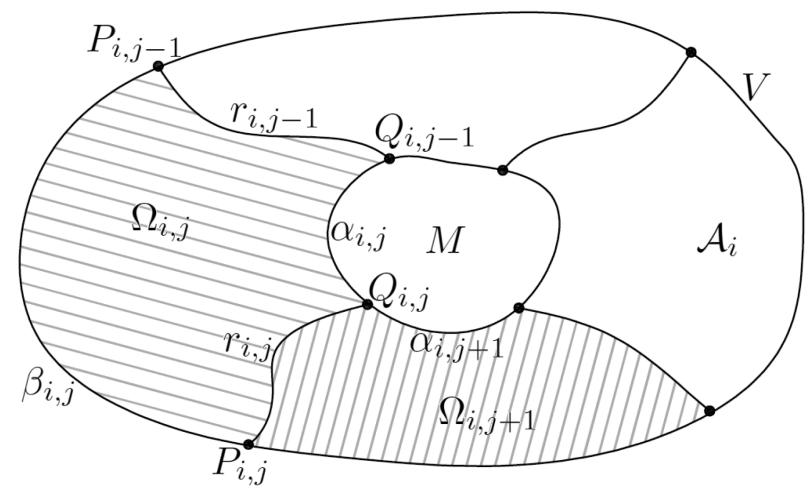

Figure 3.3. The annulus $\mathcal{A}_{i} \subset V-M^{\circ}$

The first deformation stage starts with the following.

Claim 3.3. There exists a generalized null curve $G: S \rightarrow \mathbb{C}^{3}$ such that

(b1) $\left.G\right|_{M}=F$,

(b2) $\left(p+L_{i, h}\right) \cap \overline{B(\delta / \mathfrak{n})}=\emptyset, \forall p \in \bigcup_{\mathfrak{v} \in \mathfrak{Z}} G^{\mathfrak{v}}\left(r_{i, j}\right), \forall h \in\{j, j+1\}, \forall i, j$, and

(b3) $\left(G^{\mathfrak{v}}\left(P_{i, j}\right)+L_{i, h}\right) \cap \overline{B(\kappa)}=\emptyset, \forall \mathfrak{v} \in \mathfrak{Z}, \forall h \in\{j, j+1\}, \forall i, j$. 
Proof. Write $F=\left(F_{1}, F_{2}, F_{3}\right)$ and for each $i, j$ set

$d_{i, j}(t)=\left(t F_{1}\left(Q_{i, j}\right), t F_{2}\left(Q_{i, j}\right), F_{3}\left(Q_{i, j}\right)+(t-1) \imath \sqrt{F_{1}\left(Q_{i, j}\right)^{2}+F_{2}\left(Q_{i, j}\right)^{2}}\right), t \geq 1$.

From (3.1) one has $\left(\left|F_{1}\right|+\left|F_{2}\right|\right)\left(Q_{i, j}\right) \neq 0$, and so $d_{i, j}$ is a real half-line in $\mathbb{C}^{3}$ satisfying $\prec d_{i, j}^{\prime}(t), d_{i, j}^{\prime}(t) \succ=0$. Moreover, $d_{i, j}(t)^{\mathfrak{v}}=t F^{\mathfrak{v}}\left(Q_{i, j}\right)$ for all $t \geq 1$ and $\mathfrak{v} \in \mathfrak{Z}$. Since $\left(F^{\mathfrak{v}}\left(Q_{i, j}\right)+L_{i, h}\right) \cap \overline{B(\delta / \mathfrak{n})}=\emptyset$ (see (3.2) and (a3)), the vector $F^{\mathfrak{v}}\left(Q_{i, j}\right)$ points to the connected component of $\mathbb{R}^{2}-\left(F^{\mathfrak{v}}\left(Q_{i, j}\right)+L_{i, h}\right)$ disjoint from $\overline{B(\delta / \mathfrak{n})}$, and so $\left(d_{i, j}(t)^{\mathfrak{v}}+L_{i, h}\right) \cap \overline{B(\delta / \mathfrak{n})}=\emptyset$ for all $t \geq 1, h \in\{j, j+1\}$ and $\mathfrak{v} \in \mathfrak{Z}$. Furthermore, we can take $t_{0}>1$ so that $\left(d_{i, j}\left(t_{0}\right)^{\mathfrak{v}}+L_{i, h}\right) \cap \overline{B(\kappa)}=\emptyset$ for all $\mathfrak{v} \in \mathfrak{Z}$ and $h \in\{j, j+1\}$. Up to a slight smoothing around the points $Q_{i, j}$ for all $i, j$, it suffices to set $G\left(r_{i, j}\right)=d_{i, j}\left(\left[1, t_{0}\right]\right)$ for all $i, j$ and $\left.G\right|_{M}=F$.

Then Lemma 2.6 applied to $G$ straightforwardly provides a non-flat $H \in \mathrm{N}(V)$ satisfying that

(c1) $\|H-F\|_{1, M}<\epsilon /(k m+1)$,

(c2) $\left(p+L_{i, h}\right) \cap \overline{B(\delta / \mathfrak{n})}=\emptyset, \forall p \in \bigcup_{\mathfrak{v} \in \mathfrak{Z}} H^{\mathfrak{v}}\left(r_{i, j}\right), \forall h \in\{j, j+1\}, \forall i, j$,

(c3) $\left(H^{\mathfrak{v}}\left(P_{i, j}\right)+L_{i, h}\right) \cap \overline{B(\kappa)}=\emptyset, \forall \mathfrak{v} \in \mathfrak{Z}, \forall h \in\{j, j+1\}, \forall i, j$, and

(c4) $H^{\mathfrak{Z}}\left(\alpha_{i, j}\right) \subset U_{i, j} \in \mathcal{U}$ for all $i$ and $j$.

Roughly speaking, properties (c1), (c2), and (c3) mean that $H$ satisfies (L1), (L2), and (L3) just on $S$, respectively.

Denote by $\Omega_{i, j}$ the closed disc in $\mathcal{A}_{i}$ bounded by $\alpha_{i, j} \cup r_{i, j-1} \cup r_{i, j}$ and a piece, named $\beta_{i, j}$, of $\beta_{i}$ connecting $P_{i, j-1}$ and $P_{i, j}$. Obviously $\Omega_{i, j} \cap \Omega_{i, j+1}=r_{i, j} \forall i, j$, and $\mathcal{A}_{i}=\bigcup_{j=1}^{m} \Omega_{i, j}$ for all $i$. See Figure 3.3.

Let $\eta:\{1, \ldots, k m\} \rightarrow\{1, \ldots, k\} \times \mathbb{Z}_{m}$ be the bijection $\eta(n)=\left(\mathcal{E}\left(\frac{n-1}{m}\right)+1, n-1\right)$, where $\mathcal{E}(\cdot)$ means the integer part.

The second deformation process is included in the following.

Claim 3.4. There exists a sequence $H_{0}=H, H_{1}, \ldots, H_{k m}$ of non-flat null curves in $\mathrm{N}(V)$ such that

$\left(\mathrm{d} 1_{n}\right)\left\|H_{n}-H_{n-1}\right\|_{1, \overline{V-\Omega_{\eta(n)}}}<\epsilon /(k m+1), n \geq 1$,

$\left(\mathrm{d} 2_{n}\right)\left(p+L_{h}\right) \cap \overline{B(\delta / \mathfrak{n})}=\emptyset, \forall p \in \bigcup_{\mathfrak{v} \in \mathfrak{Z}} H_{n}^{\mathfrak{v}}\left(r_{\eta(a)}\right), \forall h \in\{\eta(a), \eta(a)+(0,1)\}$, $\forall a \in\{1, \ldots, k m\}$,

$\left(\mathrm{d} 3_{n}\right)\left(H_{n}^{\mathfrak{v}}\left(P_{\eta(a)}\right)+L_{h}\right) \cap \overline{B(\kappa)}=\emptyset, \forall \mathfrak{v} \in \mathfrak{Z}, \forall h \in\{\eta(a), \eta(a)+(0,1)\}, \forall a \in$ $\{1, \ldots, k m\}$,

$\left(\mathrm{d}_{n}\right) H_{n}^{\mathfrak{Z}}\left(\alpha_{\eta(a)}\right) \subset U_{\eta(a)}, \forall a \in\{1, \ldots, k m\}$,

$\left(\mathrm{d} 5_{n}\right) \llbracket H_{n}^{\mathfrak{Z}} \rrbracket_{\Omega_{\eta(a)}}>\delta / \mathfrak{n}$ for all $a \in\{1, \ldots, n\}, n \geq 1$, and

$\left(\mathrm{d} 6_{n}\right) \llbracket H_{n}^{\mathfrak{Z}} \rrbracket_{\beta_{\eta(a)}}>\kappa$ for all $a \in\{1, \ldots, n\}, n \geq 1$.

Now, properties $\left(\mathrm{d} 1_{n}\right),\left(\mathrm{d} 5_{n}\right)$, and $\left(\mathrm{d} 6_{n}\right)$ imply that $H_{n}$ formally satisfies (L1), (L2), and (L3) on $M \cup\left(\bigcup_{a=1}^{n} \Omega_{\eta(a)}\right)$. In particular, $H_{k m}$ will solve Lemma 3.1 .

Proof of Claim 3.4. From (c2), (c3), and (c4), $H_{0}=H$ satisfies (d2 $\left.2_{0}\right),\left(\mathrm{d} 3_{0}\right)$, and $\left(\mathrm{d} 4_{0}\right)$, whereas the remaining properties make no sense for $n=0$. Reason by induction and assume that we already have $H_{0}, \ldots, H_{n-1}, n \geq 1$, satisfying the corresponding properties. Let us construct $H_{n}$.

For any $\nu=(x, y) \in \mathbb{R}^{2}-\{(0,0)\}$, it is clear that $e=(x, x, y, y, 0,0) \equiv$ $(1+\imath)(x, y, 0)$ is a non-null vector in $\mathbb{C}^{3}$ and $\ll e \gg^{\perp}=\left\{u \in \mathbb{C}^{3} \mid\left\langle u^{\mathfrak{v}}, \nu\right\rangle=0 \forall \mathfrak{v} \in \mathbb{S}^{1}\right\}$ (here $\langle\cdot, \cdot\rangle$ denotes the inner product in $\mathbb{R}^{2}$ ). 
In particular, if $\nu_{n}=\left(x_{n}, y_{n}\right) \in \mathbb{R}^{2}$ is a unit normal vector to $L_{\eta(n)}, e_{n}:=$ $(1+\imath)\left(x_{n}, y_{n}, 0\right)$, and $w_{n}:=\overline{e_{n}} / \prec \overline{e_{n}}, \overline{e_{n}} \succ$, one has

$$
\prec w_{n} \succ^{\perp}=\ll e_{n} \gg^{\perp} \subset\left\{u \in \mathbb{C}^{3} \mid\left\langle u^{\mathfrak{v}}, \nu_{n}\right\rangle=0 \forall \mathfrak{v} \in \mathfrak{Z}\right\} .
$$

(The inclusion in (3.3) becomes an equality when $\mathfrak{Z} \nsubseteq\{\mathfrak{v},-\mathfrak{v}\}$ for all $\mathfrak{v} \in \mathbb{S}^{1}$.)

Since $w_{n}$ is not null, we can take $u_{n}, v_{n} \in \prec w_{n} \succ^{\perp}$ so that $\left\{u_{n}, v_{n}, w_{n}\right\}$ is a $\prec \cdot, \succ$-conjugate basis of $\mathbb{C}^{3}$. Consider the complex orthogonal matrix $A_{n}=$ $\left(u_{n}, v_{n}, w_{n}\right)^{-1}$, define $G_{n}:=A_{n} \circ H_{n-1} \in \mathrm{N}(V)$, and write $G_{n}=\left(G_{n, 1}, G_{n, 2}, G_{n, 3}\right)$. Notice that

$$
A_{n}\left(\ll e_{n} \gg^{\perp}\right)=\left\{\left(z_{1}, z_{2}, 0\right) \in \mathbb{C}^{3} \mid z_{1}, z_{2} \in \mathbb{C}\right\} .
$$

Choose a closed disc $K_{n}$ in $\Omega_{\eta(n)}-\left(r_{\eta(n)-(0,1)} \cup \alpha_{\eta(n)} \cup r_{\eta(n)}\right)$ such that

(e1) $K_{n} \cap \beta_{\eta(n)}$ is a Jordan arc,

(e2) $\left(p+L_{\eta(n)}\right) \cap \overline{B(\delta / \mathfrak{n})}=\emptyset, \forall p \in \bigcup_{\mathfrak{v} \in \mathfrak{Z}} H_{n-1}^{\mathfrak{v}}\left(\overline{\Omega_{\eta(n)}-K_{n}}\right)$, and

(e3) $\left(p+L_{\eta(n)}\right) \cap \overline{B(\kappa)}=\emptyset, \forall p \in \bigcup_{\mathfrak{v} \in \mathfrak{Z}} H_{n-1}^{\mathfrak{v}}\left(\overline{\beta_{\eta(n)}-K_{n}}\right)$.

This choice can be guaranteed by a continuity argument. Property (e2) follows from $\left(\mathrm{d} 2_{n-1}\right),\left(\mathrm{d} 4_{n-1}\right)$, and (3.2), whereas (e3) follows from $\left(\mathrm{d} 3_{n-1}\right)$.

Now consider a Jordan arc $\gamma_{n} \subset \overline{\Omega_{\eta(n)}-K_{n}}$ with endpoints $R_{n} \in \alpha_{\eta(n)}-$ $\left\{Q_{\eta(n)-(0,1)}, Q_{\eta(n)}\right\}$ and $T_{n} \in\left(\partial K_{n}\right)-\beta_{\eta(n)}$, and otherwise disjoint from $K_{n} \cup$ $\left(\partial \Omega_{\eta(n)}\right)$ (see Figure 3.4). Without loss of generality, assume that $K_{n}$ and $\gamma_{n}$ are

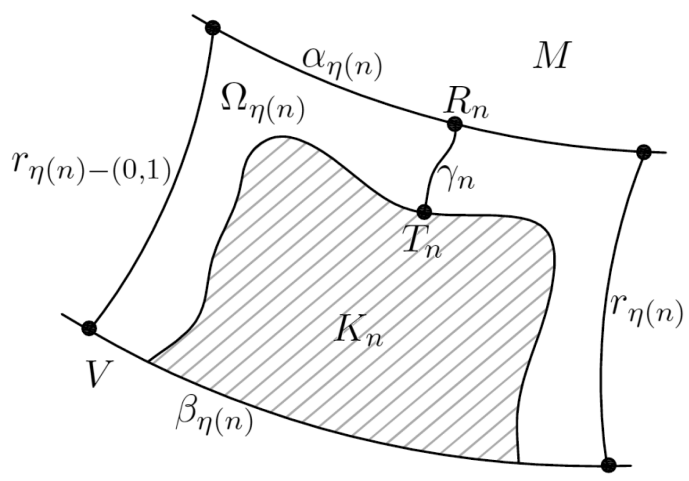

FIGURE 3.4. The closed disc $\Omega_{\eta(n)}$

chosen so that the compact set $S_{n}:=\left(\overline{V-\Omega_{\eta(n)}}\right) \cup K_{n} \cup \gamma_{n}$ is admissible and $\left.\left(d G_{n, 3}\right)\right|_{\gamma_{n}}$ never vanishes (recall that $H_{n-1}$ is non-flat and therefore so is $\left.G_{n}\right)$.

Consider $\mathfrak{v}_{0} \in \mathbb{S}^{1} \subset \mathbb{C}$ such that $\operatorname{Re}\left(\mathfrak{v} \mathfrak{v}_{0}\right) \neq 0 \forall \mathfrak{v} \in \mathfrak{Z}$, denote $\mu=\mathfrak{v}_{0}\left(y_{n},-x_{n}, \imath\right)$ $\in \mathbb{C}^{3}$, and observe that $\mu^{\mathfrak{v}}=\operatorname{Re}\left(\mathfrak{v}_{0}\right)\left(y_{n},-x_{n}\right) \neq(0,0)$ for all $\mathfrak{v} \in \mathfrak{Z}$. Therefore, there exists a large enough $C_{n}>0$ such that

$$
\llbracket\left(\mu_{n}+H_{n-1}\right)^{\mathfrak{3}} \rrbracket_{K_{n}}>\kappa,
$$

where $\mu_{n}=C_{n} \mu$. Notice also that $\mu_{n} \in \Theta \cap \ll e_{n} \gg^{\perp}$.

Denote $\zeta=A_{n}\left(\mu_{n}\right) \in \Theta \cap A_{n}\left(\ll e_{n} \gg^{\perp}\right)$. Taking into account (3.4), there exists a null vector $\zeta^{*} \in A_{n}\left(\ll e_{n} \gg^{\perp}\right)$ so that $\left\{\zeta, \zeta^{*}\right\}$ is a basis of $A_{n}\left(\ll e_{n} \gg^{\perp}\right)$ and $\prec \zeta, \zeta^{*} \succ \neq 0$.

Let $\gamma_{n}(u), u \in[0,1]$, be a smooth parameterization of $\gamma_{n}$ with $\gamma_{n}(0)=R_{n}$. Label $\tau_{j}=\gamma_{n}([0,1 / j])$ and consider the parameterization $\tau_{j}(u)=\gamma_{n}(u / j), u \in[0,1]$. 
Write $Y_{j}(u)=G_{n, 3}\left(\tau_{j}(u)\right), u \in[0,1]$, and notice that $\frac{d Y_{j}}{d u}(0)=\frac{1}{j} \frac{d\left(G_{n, 3} \circ \gamma_{n}\right)}{d u}(0) \neq 0$ for all $j \in \mathbb{N}$. Set $\zeta_{j}=\zeta-\frac{\left(d Y_{j} / d u(0)\right)^{2}}{2 \prec \zeta, \zeta^{*} \succ} \zeta^{*}, j \in \mathbb{N}$, and observe that $\lim _{j \rightarrow \infty} \zeta_{j}=\zeta$ and $\prec \zeta_{j}, \zeta_{j} \succ=-\left(\frac{d Y_{j}}{d u}(0)\right)^{2} \neq 0$ for all $j$.

Set $h_{j}:[0,1] \rightarrow \mathbb{C}^{3}$,

$$
h_{j}(u)=G_{n}\left(R_{n}\right)+\imath \frac{Y_{j}(u)-Y_{j}(0)}{\prec \zeta_{j}, \zeta_{j} \succ^{1 / 2}} \zeta_{j}+\left(0,0, Y_{j}(u)-G_{n, 3}\left(R_{n}\right)\right) .
$$

Since $\zeta_{j} \in A_{n}\left(\ll e_{n} \gg^{\perp}\right)$, then $\prec h_{j}^{\prime}(u), h_{j}^{\prime}(u) \succ=0$ and $\ll h_{j}^{\prime}(u), h_{j}^{\prime}(u) \gg$ never vanishes on $[0,1], j \in \mathbb{N}$; see (3.4). Up to choosing a suitable branch of $\prec \zeta_{j}, \zeta_{j} \succ^{1 / 2}$, the sequence $\left\{h_{j}\right\}_{j \in \mathbb{N}}$ converges uniformly on $[0,1]$ to $h_{\infty}:[0,1] \rightarrow \mathbb{C}^{3}$,

$$
h_{\infty}(u)=u \zeta+G_{n}\left(R_{n}\right) .
$$

On the other hand $\left\{\tau_{j}(1)\right\}_{j \in \mathbb{N}} \rightarrow R_{n}$, and so $\left\{h_{j}(1)-G_{n}\left(\tau_{j}(1)\right)\right\}_{j \in \mathbb{N}} \rightarrow \zeta=$ $A_{n}\left(\mu_{n}\right)$. Taking into account (3.5), there exists a large enough $j_{0} \in \mathbb{N}$ such that

$$
\llbracket\left(A_{n}^{-1}\left(h_{j_{0}}(1)-G_{n}\left(\tau_{j_{0}}(1)\right)\right)+H_{n-1}\right)^{\mathfrak{3}} \rrbracket_{K_{n}}>\kappa .
$$

Set $\hat{h}: \tau_{j_{0}}([0,1]) \rightarrow \mathbb{C}^{3}, \hat{h}(P)=h_{j_{0}}\left(\tau_{j_{0}}^{-1}(P)\right)$. Identify $\tau_{j_{0}} \equiv \tau_{j_{0}}([0,1])$, and denote by $\hat{G}_{n}=\left(\hat{G}_{n, 1}, \hat{G}_{n, 2}, \hat{G}_{n, 3}\right): S_{n} \rightarrow \mathbb{C}^{3}$ the continuous map given by

$$
\begin{aligned}
\left.\hat{G}_{n}\right|_{\overline{V-\Omega_{\eta(n)}}}= & \left.G_{n}\right|_{\overline{V-\Omega_{\eta(n)}}},\left.\hat{G}_{n}\right|_{\tau_{j_{0}}}=\hat{h}, \\
& \left.\hat{G}_{n}\right|_{\left(\gamma_{n}-\tau_{j_{0}}\right) \cup K_{n}}=\left.G_{n}\right|_{\left(\gamma_{n}-\tau_{j_{0}}\right) \cup K_{n}}-G_{n}\left(\tau_{j_{0}}(1)\right)+\hat{h}\left(\tau_{j_{0}}(1)\right) .
\end{aligned}
$$

Notice that $\hat{G}_{n, 3}=\left.\left(G_{n, 3}\right)\right|_{S_{n}}$. The equation $\prec d \hat{G}_{n}, d \hat{G}_{n} \succ=0$ formally holds except at the points $R_{n}$ and $\tau_{j_{0}}(1)$ where smoothness could fail. Up to smooth approximation (only affecting $\hat{G}_{n, 1}$ and $\hat{G}_{n, 2}$ ), $\hat{G}_{n}$ is a generalized null curve satisfying that

$$
\left.\hat{G}_{n}\right|_{\overline{V-\Omega_{\eta(n)}}}=\left.G_{n}\right|_{\overline{V-\Omega_{\eta(n)}}}, \hat{G}_{n, 3}=\left.G_{n, 3}\right|_{S_{n}}, \llbracket\left(A_{n}^{-1} \circ \hat{G}_{n}\right)^{\mathfrak{3}} \rrbracket_{K_{n}}>\kappa .
$$

Here we have taken into account (3.6), (3.7), and $H_{n-1}=A_{n}^{-1} \circ G_{n}$. Applying Lemma 2.6 to $\hat{G}_{n}$ and $S_{n}$ we can find $Z_{n}=\left(Z_{n, 1}, Z_{n, 2}, Z_{n, 3}\right) \in \mathrm{N}(V)$ so that

- $\left\|Z_{n}-\hat{G}_{n}\right\|_{1, \overline{V-\Omega_{\eta(n)}}}<\epsilon_{0}$, where $\epsilon_{0}>0$ will be specified later,

- $Z_{n, 3}=G_{n, 3}$ on $V$, and

- $\llbracket\left(A_{n}^{-1} \circ Z_{n}\right)^{\mathfrak{3}} \rrbracket_{K_{n}}>\kappa$.

Set $H_{n}:=A_{n}^{-1} \circ Z_{n} \in \mathrm{N}(V)$, and let us rewrite these properties in terms of $H_{n}$ and $H_{n-1}$ (recall that $\left.G_{n}=A_{n} \circ H_{n-1}\right)$ :

(f1) $\left\|H_{n}-H_{n-1}\right\|_{1, \overline{V-\Omega_{\eta(n)}}}<\epsilon_{0} \cdot\left\|A_{n}^{-1}\right\|($ see (3.8) $)$,

(f2) $\ll H_{n}-H_{n-1}, e_{n} \gg=0$ on $V$ (see (3.4)), and

(f3) $\llbracket H_{n}^{\mathfrak{Z}} \rrbracket_{K_{n}}>\kappa$.

To finish, let us check that $H_{n}$ satisfies the required properties provided that $\epsilon_{0}$ is small enough. Indeed, (f1) directly gives $\left(\mathrm{d} 1_{n}\right)$ if $\epsilon_{0}<\frac{\epsilon}{\left\|A_{n}^{-1}\right\|(1+k m)}$. Moreover, $\left(\mathrm{d} 2_{n}\right)$ (respectively, $\left(\mathrm{d} 3_{n}\right),\left(\mathrm{d} 4_{n}\right)$ ) follows from $(\mathrm{f} 1)$ and $\left(\mathrm{d} 2_{n-1}\right)$ (respectively, $\left(\mathrm{d} 3_{n-1}\right)$, $\left.\left(\mathrm{d} 4_{n-1}\right)\right)$ for a small enough $\epsilon_{0}$.

To check $\left(\mathrm{d} 5_{n}\right)$ we distinguish two cases. If $a<n$ (and so $n>1$ ), then we finish by using $\left(\mathrm{d} 5_{n-1}\right)$ and (f1) for a small enough $\epsilon_{0}$. In case $a=n$ we argue as 
follows. Assume first that $P \in \Omega_{\eta(n)}-K_{n}$. Then (f2) gives that $H_{n}(P)-H_{n-1}(P) \in$ $\ll e_{n} \gg^{\perp}$, and so, by (3.3),$\left\langle H_{n}^{\mathfrak{v}}(P)-H_{n-1}^{\mathfrak{v}}(P), \nu_{n}\right\rangle=0 \forall \mathfrak{v} \in \mathfrak{Z}$. Write $H_{n}^{\mathfrak{v}}(P)=$ $H_{n-1}^{\mathfrak{v}}(P)+\left(H_{n}^{\mathfrak{v}}(P)-H_{n-1}^{\mathfrak{v}}(P)\right)$, and notice that $H_{n-1}^{\mathfrak{v}}(P) \in H_{n-1}^{\mathfrak{v}}\left(\overline{\Omega_{\eta(n)}-K_{n}}\right)$ and $H_{n}^{\mathfrak{v}}(P)-H_{n-1}^{\mathfrak{v}}(P) \in L_{\eta(n)}, \forall \mathfrak{v} \in \mathfrak{Z}$. By $(\mathrm{e} 2)$ we infer that $\llbracket H_{n}^{\mathfrak{Z}} \rrbracket(P)>\delta / \mathfrak{n}$ and we are done. Assume now that $P \in K_{n}$. In this case, (f3) directly gives that $\llbracket H_{n}^{\mathfrak{Z}} \rrbracket(P)>\kappa>\delta / \mathfrak{n}$ as well.

The proof of $\left(d 6_{n}\right)$ is analogous to that of $\left(d 5_{n}\right)$. In case $a<n$, we use $\left(d 6_{n-1}\right)$ and (f1) for small enough $\epsilon_{0}$. In case $a=n$, we argue as in the proof of $\left(\mathrm{d} 5_{n}\right)$ but using (e3) instead of (e2). In this case we get that $\llbracket H_{n}^{\mathfrak{Z}} \rrbracket(P)>\kappa$ for all $P \in \beta_{\eta(n)}-K_{n}$. Finally, (f3) shows that $\llbracket H_{n}^{\mathfrak{Z}} \rrbracket(P)>\kappa$ for all $P \in K_{n}$.

The proof of Claim 3.4 is done.

Set $\hat{F}:=H_{k m} \in \mathrm{N}(V)$. Properties $(\mathrm{c} 1)$ and $\left(\mathrm{d} 1_{n}\right), n=1, \ldots, k m$, imply (L1), whereas properties (L2) and (L3) directly follow from $\left(\mathrm{d} 5_{k m}\right)$ and $\left(\mathrm{d} 6_{k m}\right)$, respectively. Therefore, $\hat{F}$ solves the basis of the induction.

3.2. Inductive step. Let $n \in \mathbb{N}$, assume that Lemma 3.1 holds when $-\chi\left(V-M^{\circ}\right)$ $<n$, and let us show that it also holds when $-\chi\left(V-M^{\circ}\right)=n$.

Recall that $M$ is admissible, and so $\mathcal{H}_{1}(M, \mathbb{Z}) \subset \mathcal{H}_{1}(V, \mathbb{Z})$. Since $-\chi\left(V-M^{\circ}\right)=$ $n>0$, there exists a Jordan curve $\hat{\gamma} \subset V^{\circ}$ intersecting $V^{\circ}-M^{\circ}$ in a Jordan arc $\gamma$ with endpoints $P, Q \in \partial M$ and otherwise disjoint from $\partial M$, and such that $\hat{\gamma} \in \mathcal{H}_{1}(V, \mathbb{Z})-\mathcal{H}_{1}(M, \mathbb{Z})$. Consequently, since $V$ is admissible, $\hat{\gamma}$ can be chosen so that $S:=M \cup \gamma$ is admissible as well.

At this point, we need the following.

Claim 3.5. The set $\Sigma=\left\{u \in \mathbb{C}^{2} \mid\|\operatorname{Re}(\mathfrak{v} u)\|>\delta \forall \mathfrak{v} \in \mathfrak{Z}\right\}$ is path-connected.

Furthermore, given $v, w$ in $\Sigma \times \mathbb{C}$ there exists a smooth arc $c:[0,1] \rightarrow \Sigma \times \mathbb{C}$ such that $c(0)=v, c(1)=w$ and $c^{\prime}(t) \in \Theta \forall t \in[0,1]$.

Proof. Fix two different points $u_{1}, u_{2} \in \Sigma$. Notice that $\ell_{u}:=\{t u \mid t>1\} \subset \Sigma$ for all $u \in \Sigma$.

Denote by $\mathbb{S}^{3}(R)$ the 3 -dimensional Euclidean sphere of radius $R>0$ in $\mathbb{R}^{4} \equiv \mathbb{C}^{2}$ and write $\mathbb{S}^{3} \equiv \mathbb{S}^{3}(1)$.

For each $\mathfrak{v} \in \mathfrak{Z}$, let $\gamma_{\mathfrak{v}} \subset \mathbb{S}^{3} \equiv \mathbb{S}^{3}(1)$ denote the spherical geodesic $H_{\mathfrak{v}} \cap \mathbb{S}^{3}$, where $H_{\mathfrak{v}}=\left\{u \in \mathbb{C}^{2} \mid \operatorname{Re}(\mathfrak{v} u)=0\right\}$, and denote $\Gamma=\bigcup_{\mathfrak{v} \in \mathfrak{3}} \gamma_{\mathfrak{v}}$. Notice that $u_{i} /\left\|u_{i}\right\| \notin \Gamma$ $\forall i=1,2$. Since $\frac{1}{R}\left(\mathbb{S}^{3}(R)-\Sigma\right)$ is the tubular neighborhood of $\Gamma$ in $\mathbb{S}^{3}$ given by $\bigcup_{\mathfrak{v} \in \mathfrak{Z}}\left\{u \in \mathbb{S}^{3} \mid\|\operatorname{Re}(\mathfrak{v} u)\| \leq \delta / R\right\}$, one has

$$
\lim _{R \rightarrow+\infty} \frac{1}{R}\left(\mathbb{S}^{3}(R)-\Sigma\right)=\Gamma
$$

in the topology associated to the Hausdorff distance. Since $\mathbb{S}^{3}-\Gamma$ is path-connected and contains $u_{1} /\left\|u_{1}\right\|$ and $u_{2} /\left\|u_{2}\right\|$, then these two points lie in the same connected component of $\frac{1}{R}\left(\mathbb{S}^{3}(R) \cap \Sigma\right)$ for a large enough $R$. Equivalently, $\frac{R}{\left\|u_{1}\right\|} u_{1}$ and $\frac{R}{\left\|u_{2}\right\|} u_{2}$ lie in the same path-connected component $\Omega$ of $\mathbb{S}^{3}(R) \cap \Sigma$. Then, $\ell_{u_{1}} \cup \Omega \cup \ell_{u_{2}}$ is path-connected and so is $\Sigma$.

For the second part, since $\Sigma$ is open and path-connected, then there exists a polygonal arc $\hat{c}:[0,1] \rightarrow \Sigma \times \mathbb{C}$ connecting $v$ and $w$ and with $\hat{c}^{\prime}(t) \in \Theta$ at any regular point. To finish, choose $c$ as a suitable smoothing of $\hat{c}$. 
By Claim 3.5 and (3.1), one can construct a generalized null curve $G: S \rightarrow \mathbb{C}^{3}$ satisfying $\left.G\right|_{M}=F$ and $\llbracket G^{\mathfrak{3}} \rrbracket_{\gamma}>\delta$. From Lemma 2.6 applied to $G$ and a continuity argument, we obtain a compact region $U$ and a null curve $H \in \mathrm{N}(U)$ satisfying that

(i) $S \subset U^{\circ} \subset U \subset V^{\circ}$,

(ii) $-\chi\left(V-U^{\circ}\right)=n-1$,

(iii) $\|H-G\|_{1, M}<\epsilon / 2$, and

(iv) $\llbracket H^{\mathfrak{3}} \rrbracket_{U-M^{\circ}}>\delta$.

Since $-\chi\left(V-U^{\circ}\right)<n$, the induction hypothesis applied to $H$ and $\epsilon_{0}=$ $\min \{\epsilon / 2, \delta-\delta / \mathfrak{n}\}$ gives a null curve $\hat{F} \in \mathrm{N}(V)$ such that

(I) $\|\hat{F}-H\|_{1, U}<\epsilon_{0}$,

(II) $\llbracket \hat{F}^{\mathfrak{3}} \rrbracket_{V-U^{\circ}}>\delta / \mathfrak{n}$, and

(III) $\llbracket \hat{F}^{3} \rrbracket \partial V>\kappa$.

Then, (L1) follows from (iii) and (I). Properties (iv), (I), and (II) give (L2). Finally, (III) directly implies (L3). Hence, $\hat{F}$ satisfies the conclusion of Lemma 3.1 and we are done.

\section{Main Results}

Given an admissible compact region $M \subset \mathcal{N}, F \in \mathrm{N}(M)$, a finite subset $\mathfrak{Z} \subset \mathbb{S}^{1}$ and $r>0$, it is not hard to find $v \in \mathbb{C}^{3}$ so that the null curve $X=F+v$ satisfies that $\llbracket X^{\mathfrak{3}} \rrbracket_{\partial M}>r$. Indeed, it suffices to choose $v \in \mathbb{C}^{3}$ such that $\|F\|<\left\|v^{\mathfrak{v}}\right\|-r$ on $M$ for all $\mathfrak{v} \in \mathfrak{Z}$. Therefore, the Main Theorem in the introduction follows from the following extension result.

Theorem 4.1. Let $M$ be an admissible compact region in $\mathcal{N}$, let $\mathfrak{Z}_{0} \subset \mathbb{S}^{1}$ be a finite subset with cardinal number $\mathfrak{n} \in \mathbb{N}$, and let $X \in \mathrm{N}(M)$ such that

$$
\llbracket X^{\mathfrak{Z}_{0}} \rrbracket_{\partial M}>\mathfrak{n} .
$$

Then, for any $\varepsilon>0$, there exist an infinite closed subset $\mathfrak{Z}_{\mathcal{N}} \subset \mathbb{S}^{1}$ and $Y \in \mathrm{N}(\mathcal{N})$ such that

(A) $\mathfrak{Z}_{0} \subset \mathfrak{Z}_{\mathcal{N}}$,

(B) $\|Y-X\|_{1, M}<\varepsilon$,

(C) the map $\mathfrak{Y}: \mathfrak{Z}_{\mathcal{N}} \times \mathcal{N} \rightarrow \mathbb{R}^{2}$ given by $\mathfrak{Y}(\mathfrak{v}, P)=Y^{\mathfrak{v}}(P)$ is proper, and

(D) $\llbracket Y^{30} \rrbracket>1-\varepsilon$ on $\mathcal{N}-M$.

Proof. Without loss of generality, we assume that $\varepsilon<1$.

Let $\left\{M_{n} \mid n \in\{0\} \cup \mathbb{N}\right\}$ be an exhaustion of $\mathcal{N}$ by admissible compact regions with analytical boundary satisfying that $M_{0}=M$ and $M_{n-1} \subset M_{n}^{\circ}$ for all $n \in \mathbb{N}$.

Label $X_{0}=X$ and let us construct a sequence $\left\{\left(X_{n}, \mathfrak{Z}_{n}\right)\right\}_{n \in \mathbb{N}}$ of null curves and finite subsets satisfying that

$\left(\mathrm{a}_{n}\right) X_{n} \in \mathrm{N}\left(M_{n}\right)$ for all $n \in \mathbb{N}$,

$\left(b_{n}\right) \mathfrak{Z}_{n-1} \subset \mathfrak{Z}_{n} \subset \mathbb{S}^{1}$ and the cardinal number of $\mathfrak{Z}_{n}$ is $\mathfrak{n}+n$,

(c $\left.c_{n}\right)\left\|X_{n}-X_{n-1}\right\|_{1, M_{n-1}}<\varepsilon_{n}$ for all $n \in \mathbb{N}$, where

$$
\varepsilon_{n}<\frac{1}{2^{n+1}} \min \left\{\varepsilon, \min \left\{\min _{M_{k}}\left\|\frac{d X_{k}}{\sigma_{\mathcal{N}}}\right\| \mid k=0, \ldots, n-1\right\}\right\}>0
$$

(notice that $d X_{k}$ never vanishes on $M_{k}$ since $X_{k} \in \mathrm{N}\left(M_{k}\right)$ ),

$\left(\mathrm{d}_{n}\right) \llbracket X_{k}^{\mathfrak{Z}_{n}} \rrbracket_{M_{k}-M_{k-1}^{\circ}}>k$ for all $k \in\{1, \ldots, n\}, n \in \mathbb{N}$, and

$\left(\mathrm{e}_{n}\right) \llbracket X_{n}^{\mathfrak{Z}_{n}} \rrbracket_{\partial M_{n}}>(n+1)(\mathfrak{n}+n)$ for all $n \in \mathbb{N}$. 
The sequence is obtained in a recursive way. The couple $\left(X_{0}, \mathfrak{Z}_{0}\right)$ trivially satisfies $\left(\mathrm{a}_{0}\right)$ and $\left(\mathrm{e}_{0}\right)$, whereas $\left(\mathrm{b}_{0}\right),\left(\mathrm{c}_{0}\right)$, and $\left(\mathrm{d}_{0}\right)$ make no sense. Let $n \geq 1$, assume we already have a couple $\left(X_{n-1}, \mathfrak{Z}_{n-1}\right)$ satisfying the corresponding properties, and let us construct $\left(X_{n}, \mathfrak{Z}_{n}\right)$. For $\varepsilon_{n}$ small enough, the null curve $X_{n} \in \mathrm{N}\left(M_{n}\right)$ given by Lemma 3.1 applied to the data

$$
(M, V, \mathfrak{Z}, F, \delta, \epsilon, \kappa)=\left(M_{n-1}, M_{n}, \mathfrak{Z}_{n-1}, X_{n-1}, n(\mathfrak{n}+n-1), \varepsilon_{n},(n+1)(\mathfrak{n}+n)\right)
$$

satisfies $\left(a_{n}\right),\left(c_{n}\right)$,

$$
\llbracket X_{k}^{\mathfrak{Z}_{n-1}} \rrbracket_{M_{k}-M_{k-1}^{\circ}}>k \text { for all } k \in\{1, \ldots, n\} \text {, and } \llbracket X_{n}^{\mathfrak{Z}_{n-1}} \rrbracket_{\partial M_{n}}>(n+1)(\mathfrak{n}+n) \text {. }
$$

For the first assertion in (4.2), use items $\left(\mathrm{c}_{n}\right)$ and $\left(\mathrm{d}_{n-1}\right)$ and a continuity argument for $k \in\{1, \ldots, n-1\}$, and Lemma 3.1 (L2) for $k=n$.

To close the induction, choose any $\mathfrak{v} \in \mathbb{S}^{1}-\mathfrak{Z}_{n-1}$ such that the couple $\left(X_{n}, \mathfrak{Z}_{n}:=\right.$ $\left.\mathfrak{Z}_{n-1} \cup\{\mathfrak{v}\}\right)$ satisfies $\left(\mathrm{b}_{n}\right),\left(\mathrm{d}_{n}\right)$, and $\left(\mathrm{e}_{n}\right)$. Since $M_{k}-M_{k-1}^{\circ}, k=1, \ldots, n$, and $\partial M_{n}$ are compact, the existence of such a $\mathfrak{v}$ near $\mathfrak{Z}_{0}$ is guaranteed by a continuity argument and (4.2).

By items $\left(\mathrm{a}_{n}\right)$ and $\left(\mathrm{c}_{n}\right),\left\{X_{n}\right\}_{n \in \mathbb{N}}$ uniformly converges on compact subsets of $\mathcal{N}$ to a holomorphic map $Y: \mathcal{N} \rightarrow \mathbb{C}^{3}$ with $\prec d Y, d Y \succ=0$. Set $\mathfrak{Z}_{\infty}:=\bigcup_{n \in \mathbb{N}} \mathfrak{Z}_{n}$, $\mathfrak{Z}_{\mathcal{N}}=\overline{\mathfrak{Z}}_{\infty}$, and let us show that the couple $\left(Y, \mathfrak{Z}_{\mathcal{N}}\right)$ satisfies the theses of the theorem.

From $\left(\mathrm{b}_{n}\right), \mathfrak{Z}_{\mathcal{N}}$ is a closed infinite set. Item $(\mathrm{A})$ is obvious.

To prove that $Y$ is an immersion, hence a null curve, it suffices to check that $\left\|d Y / \sigma_{\mathcal{N}}\right\|(P)>0 \forall P \in \mathcal{N}$. Indeed, let $P \in \mathcal{N}$ and choose $j \in \mathbb{N}$ so that $P \in M_{j}$. Then $\left(\mathrm{c}_{n}\right)$ implies that

$$
\begin{aligned}
\left\|d Y / \sigma_{\mathcal{N}}\right\|(P) & \geq\left\|d X_{j} / \sigma_{\mathcal{N}}\right\|(P)-\sum_{k \geq j}\left\|X_{k+1}-X_{k}\right\|_{1, M_{k}} \\
& >\left\|d X_{j} / \sigma_{\mathcal{N}}\right\|(P)-\sum_{k \geq j} \varepsilon_{k+1} \\
& >\left\|d X_{j} / \sigma_{\mathcal{N}}\right\|(P)-\sum_{k \geq j} \frac{1}{2^{k+1}}\left\|d X_{j} / \sigma_{\mathcal{N}}\right\|(P) \\
& \geq \frac{1}{2}\left\|d X_{j} / \sigma_{\mathcal{N}}\right\|(P)>0
\end{aligned}
$$

and hence $Y$ is an immersion as claimed.

From $\left(c_{n}\right)$ one has

$$
\left\|Y-X_{j}\right\|_{1, M_{j}}<\sum_{k=j+1}^{\infty}\left\|X_{k}-X_{k-1}\right\|_{1, M_{k-1}}<\sum_{k=j+1}^{\infty} \varepsilon_{k}<\varepsilon \text { for all } j \geq 0,
$$

proving in particular (B).

To prove (C), take $k \in \mathbb{N}$. From (4.3) and $\left(\mathrm{d}_{j}\right), j \geq k$, one infers $\llbracket Y^{\mathfrak{Z}_{j}} \rrbracket_{M_{k}-M_{k-1}^{\circ}>}>$ $k-\varepsilon$ for all $j \geq k$. Therefore, $\mathfrak{Y}\left(\mathfrak{Z}_{\infty} \times\left(M_{k}-M_{k-1}^{\circ}\right)\right) \cap B(k-\varepsilon)=\emptyset$ for all $k \in \mathbb{N}$, and hence $\mathfrak{Y}^{-1}(\overline{B(k-2 \varepsilon)}) \cap\left(\mathfrak{Z}_{\infty} \times \mathcal{N}\right) \subset \mathfrak{Z}_{\infty} \times M_{k-1}$ is relatively compact in $\mathfrak{Z}_{\mathcal{N}} \times \mathcal{N}$. Thus $\mathfrak{Y}^{-1}(\overline{B(k-2 \varepsilon)})$ is compact in $\mathfrak{Z}_{\mathcal{N}} \times \mathcal{N}$ for all $k$, proving $(\mathrm{C})$.

Finally, (D) follows from $\left(\mathrm{b}_{n}\right),\left(\mathrm{d}_{n}\right)$, and (4.3). 
Remark 4.2. Theorem 4.1 shows that $\mathfrak{Z}_{0}$ is a universal projector set and $\mathfrak{Z}_{\mathcal{N}} \subset \mathbb{S}^{1}$ is a projector set for the fixed $\mathcal{N}$. Since obviously $\mathfrak{Z}_{\mathcal{N}}$ depends on $\mathcal{N}$, one cannot infer that it is a universal projector set.

On the other hand, up to an elementary refinement of the proof of Theorem 4.1, one can construct $\mathfrak{Z}_{\infty}$ to be closed, and even with accumulation set in $\mathfrak{Z}_{0}$. In this case, $\mathfrak{Z}_{\mathcal{N}}=\mathfrak{Z}_{\infty}$ is countably infinite.

The following proposition shows that $\mathbb{S}^{1}$ is a projector set for no hyperbolic Riemann surface.

Proposition 4.3. Let $M$ be an open Riemann surface and let $F=\left(F_{1}, F_{2}\right): M \rightarrow$ $\mathbb{C}^{2}$ be a holomorphic map. Assume that the map $\Psi: \mathbb{S}^{1} \times M \rightarrow \mathbb{R}^{2}, \Psi(\mathfrak{v}, P)=$ $\operatorname{Re}(\mathfrak{v} F)$, is proper.

Then $M$ is parabolic. As a consequence, if $M$ has finite topology, then $M$ is biholomorphic to a finitely punctured compact Riemann surface and $F_{1}$ and $F_{2}$ extend meromorphically to the compactification of $M$.

Proof. To show that $M$ is parabolic, it suffices to check that $F_{1}: M \rightarrow \mathbb{C}$ (and likewise $F_{2}$ ) is a proper holomorphic function. Reason by contradiction and take a divergent sequence $\left\{P_{n}\right\}_{n \in \mathbb{N}} \subset M$ such that $\left\{F_{1}\left(P_{n}\right)\right\}_{n \in \mathbb{N}}$ is bounded. For each $n \in \mathbb{N}$ choose $\mathfrak{v}_{n} \in \mathbb{S}^{1}$ such that $\operatorname{Re}\left(\mathfrak{v}_{n} F_{2}\left(P_{n}\right)\right)=0$. Then $\left\{\Psi\left(\mathfrak{v}_{n}, P_{n}\right)\right\}_{n \in \mathbb{N}}$ is bounded as well, which is absurd.

For the second part of the proposition, assume that $M$ has finite topology. The parabolicity implies that $M=\bar{M}-\left\{Q_{1}, \ldots, Q_{k}\right\}$, where $\bar{M}$ is a compact Riemann surface and $Q_{1}, \ldots, Q_{k} \in \bar{M}$. Since $F_{1}, F_{2}: M \rightarrow \mathbb{C}$ are proper holomorphic functions, they have no essential singularities at the ends, and so they extend meromorphically to $\bar{M}$.

Corollary 4.4. Let $M$ be an open Riemann surface of finite topology and let $F$ : $M \rightarrow \mathbb{C}^{3}$ be a null curve. Assume that the map $\mathfrak{Y}: \mathbb{S}^{1} \times M \rightarrow \mathbb{R}^{2}, \mathfrak{Y}(\mathfrak{v}, P)=F^{\mathfrak{v}}(P)$, is proper.

Then $F$ has finite total curvature.

Proof. Just write $F=\left(F_{j}\right)_{j=1,2,3}$, take into account that $d F_{1}$ and $d F_{2}$, hence $d F_{3}$, extend meromorphically to the natural compactification of $M$, and use Osserman's classical results $\mathrm{OS}$.

\section{REFERENCES}

[AFL] Antonio Alarcón, Isabel Fernández, and Francisco López, Complete minimal surfaces and harmonic functions, Comment. Math. Helv. 87 (2012), no. 4, 891-904, DOI 10.4171/CMH/272. MR2984575

[AL1] Antonio Alarcón and Francisco J. López, Minimal surfaces in $\mathbb{R}^{3}$ properly projecting into $\mathbb{R}^{2}$, J. Differential Geom. 90 (2012), no. 3, 351-381. MR.2916039

[AL2] A. Alarcón and F. J. López, Null curves in $\mathbb{C}^{3}$ and Calabi-Yau conjectures. Math. Ann. 355 (2013), no. 2, 429-455. MR3010135

[GN] R. C. Gunning and Raghavan Narasimhan, Immersion of open Riemann surfaces, Math. Ann. 174 (1967), 103-108. MR0223560 (36 \#6608)

[Lo] F. J. López, Uniform approximation by complete minimal surfaces of finite total curvature in $\mathbb{R}^{3}$. Trans. Amer. Math. Soc., in press.

[Mo] S. Morales, On the existence of a proper minimal surface in $\mathbb{R}^{3}$ with a conformal type of disk, Geom. Funct. Anal. 13 (2003), no. 6, 1281-1301, DOI 10.1007/s00039-003-0446-3. MR2033839 (2004m:53019)

[Os] Robert Osserman, A survey of minimal surfaces, 2nd ed., Dover Publications Inc., New York, 1986. MR852409(87j:53012) 
[Pi] Gian Pietro Pirola, Algebraic curves and non-rigid minimal surfaces in the Euclidean space, Pacific J. Math. 183 (1998), no. 2, 333-357, DOI 10.2140/pjm.1998.183.333. MR.1625966 (99e:53009)

[SY] R. Schoen and S. T. Yau, Lectures on harmonic maps, Conference Proceedings and Lecture Notes in Geometry and Topology, II, International Press, Cambridge, MA, 1997. MR1474501 (98i:58072)

Departamento de Geometría y Topología, Universidad de Granada, E-18071 Granada, SPAIN

E-mail address: alarcon@ugr.es

Departamento de Geometría y Topología, Universidad de Granada, E-18071 Granada, SPAIN

E-mail address: fjlopez@ugr.es 